\title{
IMPACTO DA PARCERIA DE CUIDADOS PARA A CRIANÇA HOSPITALIZADA E SUA FAMÍLIA
}

\author{
Rosa Maria Correia Jerónimo Pedroso \\ Professora Coordenadora \\ Escola Superior de Enfermagem de Coimbra, Portugal \\ rpedroso@esenfc.pt \\ https://doi.org/10.17060/ijodaep.2017.n1.v3.991
}

Fecha de Recepción: 22 Enero 2017

Fecha de Admisión: 1 Abril 2017

\section{RESUMO}

Antecedentes- 0 período de hospitalização representa muitas vezes para a criança 0 afastamento do seu ambiente familiar, das suas actividades diárias e dos seus amigos, pelo que a parceria de cuidados é reconhecida como essencial no seu processo de hospitalização, trazendo benefícios quer para a criança e sua família, quer para a equipa de profissionais de saúde.

Objectivo- Conhecer o impacto da parceria de cuidados de enfermagem para as crianças hospitalizadas e suas famílias.

Metodologia- Trata-se de uma Revisão Sistemática da Literatura. Após os critérios de inclusão e exclusão foram seleccionados 5 artigos primários, publicados entre 2005-2014 e pesquisados nas plataformas eletrónicas: EBSCOhost, b-on e SciELO, que deram resposta à questão de revisão.

Resultados- Os resultados obtidos demonstraram que a parceria de cuidados é benéfica tanto para a criança como para a sua família, na medida em que a oportunidade de participação nos cuidados, diminui os níveis de ansiedade, aumenta o sentimento de independência, promove uma comunicação adequada com a equipa de enfermagem, sentimentos de paz e encorajamento e facilita a adaptação à nova condição de saúde da criança.

Conclusões- Cabe à equipa de enfermagem incluir a família no processo de cuidados, permitindo a sua presença e o seu envolvimento ativo, dando a possibilidade aos familiares de prestar apoio emocional e segurança à criança hospitalizada. 0 acompanhamento ao longo de todo o processo de internamento é crucial para minimizar o impacto da hospitalização na criança e na sua família.

Palavras-chave: Parceria de cuidados, integração nos cuidados, participação nos cuidados, pais, família, pediatria e criança.

\section{ABSTRACT \\ Impact of the care partnerhip on hospitalized children and their families}




\section{IMPACTO DA PARCERIA DE CUIDADOS PARA A CRIANÇA HOSPITALIZADA E SUA FAMÍLIA}

Background - For children, hospital admission often means to be removed from their home environment, their daily activities, and their friends. The care partnership is considered to be essential in the child's hospitalization process, resulting in benefits not only for children and their families but also for healthcare professionals.

Objective - To identify the impact of the nursing care partnership on hospitalized children and their families.

Methods - A systematic literature review was conducted. After application of the inclusion/exclusion criteria, a search performed in the online databases EBSCOhost, b-on, and SciELO revealed 5 primary studies published between 2005 and 2014 that addressed the research question.

Results- The results showed that both children and their families benefit from the care partnership, to the extent that the opportunity to be involved in care delivery reduces their anxiety levels, increases their feeling of independence, promotes a good communication with the nursing team, brings about feelings of peace and reassurance, and facilitates the adaptation to the child's new health condition.

Conclusions- The nursing team is responsible for including family members in the care process, encouraging their presence and active participation and allowing them to provide emotional support and safety to the hospitalized child. Their presence throughout the hospitalization period is essential to minimize its impact on children and their families.

Keywords: Care partnership, integration into care, participation in care, parents, family, pediatrics, child.

\section{ANTECEDENTES}

0 período de hospitalização representa muitas vezes para a criança 0 afastamento do seu ambiente familiar, das suas actividades diárias e dos seus amigos, pelo que a parceria de cuidado é reconhecida como essencial no seu processo de hospitalização, trazendo benefícios quer para a criança e sua família, quer para a equipa de profissionais de saúde. Desta forma 0 ambiente hospitalar acabar por ser visto como um ambiente desconhecido e impessoal, sendo muitas vezes associado a um lugar de solidão, tristeza, saudade de casa, da escola, amigos e familiares (Valverde 2010).

0 facto de a criança passar a maior parte do tempo da hospitalização restringida ao leito e rodeada de pessoas que desconhece são considerados por Oliveira, Danilson e Dantas (2004) fatores desencadeadores de medo e sofrimento. Ainda para estes autores (Oliveira, Danilson e Dantas 2004) factores como a ansiedade e a grande variedade de sentimentos experimentados pela criança durante todo este processo, pode traduzir-se através de perturbações da alimentação, birras, alterações do sono, retrocesso dos hábitos adequados.

Lima 1985 citado por Oliveira, Danilson e Dantas 2004, defende que o processo de hospitalização acaba sempre por trazer repercussões a nível do desenvolvimento emocional da criança, podendo ser positivas ou negativas. Assim, para Oliveira (2004), as crianças que experienciam vários ou prolongados períodos de internamento, apresentam maior probabilidade de apresentarem um atraso a nível do desenvolvimento, sendo crucial a equipa de saúde ter em atenção e evitar todos os fatores que possam afetar o desenvolvimento normal da criança internada.

Uma hospitalização quer seja planeada ou não, apresenta sempre consequências a nível do quotidiano de uma família, trazendo repercussões a nível de todos os elementos da família. Assim, a hospitalização da criança, apesar das suas vantagens, gera instabilidade e um elevado desequilíbrio no sistema familiar, podendo desencadear uma situação de crise, sendo fundamental os pais serem integrados no processo de hospitalização da criança e deste modo promovendo o seu bem-estar durante o período de transição. 
0 facto de a criança se encontrar hospitalizada, coloca os pais numa situação mais vulnerável, pois existem algumas funções que passam a ser desempenhadas pelo enfermeiro durante esse período e que anteriormente eram realizadas pelos pais. Para (Santos, 2012) esta alteração pode originar nos pais um sentimento de revolta e frustração acabando por comprometer o seu papel parental, bem como as necessidades psicológicas dos familiares. 0 acompanhamento do processo de evolução da criança, a presença e a participação nos cuidados prestados à mesma, podem levar à diminuição da ansiedade sentida pelos pais, pois estes acabam por se sentir úteis durante o período de hospitalização da criança.

Segundo Cardoso 2010, (p.31), ao citar Whyte (1997) é fundamental centrar os cuidados na criança e sua família, dado a influência que este processo tem em toda a dinâmica familiar, " mais que um desenvolvimento de cuidados de enfermagem na comunidade, em pediatria ou psiquiatria, os cuidados de enfermagem à família são o desenvolvimento lógico de uma abordagem holística dos cuidados aos doentes e um compromisso na promoção da saúde.".

A aproximação da família à criança e aos cuidados que lhe são prestados, permitiram tornar 0 processo de hospitalização o menos negativo possível para os intervenientes, diminuindo os sentimentos negativos e a insegurança. É evidenciada a necessidade de permitir a participação dos pais nos cuidados de saúde e de ceder toda a informação sobre o estado de saúde da criança, de forma adaptada, para diminuir o impacto na família e consequentemente na criança (Nascimento, 2006).

Estudos realizados permitiram concluir que a aproximação dos familiares à criança, não apenas física mas também na participação nos cuidados prestados, atenua sentimentos despoletados pela hospitalização, como ansiedade, tristeza, medo, stress, sofrimento e insegurança (Idem). (Nascimento, 2006).

Ao percepcionar as relações familiares e as preocupações, o enfermeiro promove uma relação de confiança e segurança, pelo que irá mais facilmente obter uma colheita de dados o mais completa possível, permitindo-lhe em parceria com a família, planear as intervenções de enfermagem e, consequentemente a prestação de cuidados.

Anne Casey, em 1988 desenvolveu o a partir do modelo de cuidados centrados na família um modelo de cuidados pediátricos "Modelo de parceria de cuidados" que evidencia o contributo dos pais nos cuidados à criança como parceiros nos cuidados, sendo a principal finalidade satisfazer as necessidades dos pais e das crianças, com a intervenção mínima dos enfermeiros. Para que este objectivo seja concretizável, é crucial que a equipa de saúde partilhe conhecimentos e realize ensinos aos pais de modo a que estes se tornem capazes de realizar os cuidados à criança.

Deste modo, este modelo constitui uma filosofia de cuidados de enfermagem que reconhece e valoriza a importância da família para o bem-estar presente e futuro da criança.

Este modelo preconiza a não existência de fronteiras fixas entre os cuidados prestados pelos pais e os cuidados prestados pelos enfermeiros, recomendando mesmo flexibilidade nos cuidados, de forma a promover o desenvolvimento conjunto de acções e completo bem-estar da criança.

A parceria de cuidados é reconhecida como essencial no processo de hospitalização da criança, trazendo benefícios para a criança e sua família e para a equipa de profissionais de saúde. 0 conhecimento e as capacidades que os pais possuem, juntamente com as informações relativas à criança, levam a que se tornem peritos na prestação desses cuidados. Neste contexto, é crucial a comunicação estabelecida entre a equipa de saúde e os pais, onde ocorre negociação dos papéis e identificação de necessidades de apoio, passando os pais a ter um papel mais ativo em todo o processo de hospitalização, atenuando consequentemente sentimentos de insegurança, impotência e inutilidade. (Hutcthfield, 1999 e Smith et al, 2006 citados por Cardoso 2010). 


\section{IMPACTO DA PARCERIA DE CUIDADOS PARA A CRIANÇA HOSPITALIZADA E SUA FAMÍLIA}

\section{OBJECTIVO}

0 objectivo desta revisão foi conhecer os estudos acerca do impacto da parceria de cuidados de enfermagem para as crianças hospitalizadas e suas famílias.

\section{METODOLOGIA}

Foi realizada uma revisão sistemática da literatura, e que incluiu uma análise de todos os estudos relevantes identificados.

Para expressão da questão de investigação recorremos ao método PICO: Participante, Intervenções, Comparações, Outcomes (resultados).

Assim, para a actual revisão sistemática da literatura elaborou-se a seguinte questão: Em que medida a parceria de cuidados facilita/dificulta o processo de hospitalização para a criança e para a sua família?

QUADRO 1

Participantes, Intervenções, Comparações e Outcomes.

\begin{tabular}{|c|c|c|c|}
\hline $\mathbf{P}$ & $\begin{array}{l}\text { População, } \\
\text { Participantes, } \\
\text { Pacientes }\end{array}$ & $\begin{array}{l}\text { Quem foi } \\
\text { estudado? }\end{array}$ & $\begin{array}{l}\text { Crianças hospitalizadas } \\
\text { Familiares das crianças hospitalizadas }\end{array}$ \\
\hline I & $\begin{array}{l}\text { Intervenção ou } \\
\text { fenómeno de } \\
\text { Interesse }\end{array}$ & O que foi feito? & Implementação da parceria de cuidados \\
\hline $\mathbf{C}$ & $\begin{array}{l}\text { Comparação ou } \\
\text { Contexto }\end{array}$ & $\begin{array}{l}\text { Podem existir ou } \\
\text { não? }\end{array}$ & $\begin{array}{l}\text { Comparação entre o envolvimento no } \\
\text { processo de parceria de cuidados e não } \\
\text { envolvimento. }\end{array}$ \\
\hline $\mathbf{O}$ & $\begin{array}{l}\text { Outcomes, } \\
\text { resultados esperados }\end{array}$ & $\begin{array}{l}\text { Resultados/Efeitos } \\
\text { ou consequências }\end{array}$ & $\begin{array}{l}\text { Facilitar o processo de hospitalização } \\
\text { para a criança e para a sua família. }\end{array}$ \\
\hline
\end{tabular}

A pesquisa foi efetuada no período de 5 a 11 de junho de 2014, através das palavras-chave: integration care, participation care, partnership care, parent, family, pediatric e child. com um horizonte temporal de 9 anos, desde 2005 a 2014. Para a pesquisa de evidência científica foram utilizadas as seguintes plataformas eletrónicas: EBSCOhost, b-on e SciELO. Foram eliminados os artigos sem acesso gratuito a full text antes da sua leitura integral.

Para a seleção dos estudos foram definidos critérios que permitiram incluir, ou não, a evidência na revisão realizada. Desta forma, foi utilizado novamente o método de PI[C]OD: Participante, Intervenções, Comparações, Outcomes e Desenho do estudo. 


\section{CRITÉRIOS DE INCLUSÃO}

Familiares de crianças com idades compreendidas entre os 0 e os 18 anos, familiares de crianças com internamentos superiores a 48h, enfermeiros cuidadores de crianças com idades compreendidas entre os 0 e os 18 anos, estudos publicados entre 2005 e 2014, publicados em Português, Inglês, Francês e Espanhol e estudos com free full text.

\section{CRITÉRIOS DE EXCLUSÃO}

Familiares de crianças internadas em unidades de neonatologia, unidades de cuidados intensivos e serviços de urgência e Revisões sistemáticas da literatura.

\section{RESULTADOS}

Após selecionados os estudos em análise procedeu-se à sua leitura integral, considerando os critérios de seleção. Desta forma foram selecionados 5 artigos que foram analisados na íntegra e de forma independente para leitura completa e análise criteriosa, para a integração dos estudos nesta revisão sistemática da literatura.

Os estudos analisados reportam-se a uma grande variedade de desenhos de investigação, qualitativos na sua maioria (3) e qualitativos (2). Mostraram diferentes estratégias de intervenção e instrumentos de análise e o tamanho das amostras variou entre 8 e 206 participantes.

Os estudos foram realizados no Brasil (2), no Irão (1) e no Japão (2).

\section{QUADRO 2}

\section{Resumo dos artigos constituintes da Revisão Sistemática}

\begin{tabular}{|c|c|c|c|c|}
\hline $\begin{array}{l}\text { Título do } \\
\text { Estudo }\end{array}$ & Autores/País/Ano & Objectivos & $\begin{array}{l}\text { Desenho do } \\
\text { Estudo }\end{array}$ & Resultados \\
\hline $\begin{array}{l}\text { "Parents" } \\
\text { participation in } \\
\text { taking care } \\
\text { of hospitalized } \\
\text { children: A } \\
\text { concept } \\
\text { analysis with } \\
\text { hybrid model" } \\
\text { S1 - 1 }\end{array}$ & $\begin{array}{l}\text { VASLI, P.; } \\
\text { SALSALI, M.; } \\
\text { Irão (2O14) }\end{array}$ & $\begin{array}{l}\text { Definir a } \\
\text { participação dos } \\
\text { pais nos } \\
\text { cuidados à } \\
\text { criança } \\
\text { hospitalizada. }\end{array}$ & $\begin{array}{l}\text { Estudo } \\
\text { qualitativo }\end{array}$ & $\begin{array}{l}\text { Foram identificadas } \\
\text { quatro dimensões da } \\
\text { participação dos pais } \\
\text { nos cuidados à } \\
\text { criança } \\
\text { hospitalizada: a } \\
\text { cooperação com a } \\
\text { equipa de } \\
\text { enfermagem, a } \\
\text { educação e aumento } \\
\text { da conscientização } \\
\text { dos pais, a } \\
\text { habilidade no } \\
\text { atendimento e a } \\
\text { oportunidade de } \\
\text { participação e de } \\
\text { tomada de decisão. }\end{array}$ \\
\hline $\begin{array}{l}\text { "The } \\
\text { perception of } \\
\text { nursing } \\
\text { staffabout the } \\
\text { participation } \\
\text { of family in } \\
\text { care for child } \\
\text { with cancer" } \\
\mathbf{S 1}-\mathbf{2}\end{array}$ & $\begin{array}{l}\text { ANDRADE, N. } \\
\text { K.S.; } \\
\text { VALENÇA,C.; } \\
\text { LIMA,G.A.F.; } \\
\text { CAVALCANTE, } \\
\text { R. D.; } \\
\text { SALES, L.K.O.; } \\
\text { GERMANO, R. } \\
\text { M.; } \\
\text { Brasil (2012) }\end{array}$ & $\begin{array}{l}\text { Compreender a } \\
\text { perceção da } \\
\text { equipa de } \\
\text { enfermagem } \\
\text { relativa à } \\
\text { participação dos } \\
\text { familiares nos } \\
\text { cuidados à } \\
\text { criança com } \\
\text { cancro. }\end{array}$ & $\begin{array}{l}\text { Estudo } \\
\text { qualitativo, } \\
\text { exploratório } \\
\text { descritivo }\end{array}$ & $\begin{array}{l}\text { A interação com os } \\
\text { familiares através da } \\
\text { partilha de } \\
\text { informações, } \\
\text { reconhecimento de } \\
\text { soluções e o apoio de } \\
\text { ambos permite a } \\
\text { junção de recursos } \\
\text { que permitirão à } \\
\text { criança sentir-se } \\
\text { mais segura e } \\
\text { diminuir o } \\
\text { sofrimento e dor } \\
\text { causados pelo } \\
\text { processo de } \\
\text { hospitalização. }\end{array}$ \\
\hline
\end{tabular}




\begin{tabular}{|c|c|c|c|c|}
\hline $\begin{array}{l}\text { "Importância } \\
\text { da relação } \\
\text { interpessoal }\end{array}$ & $\begin{array}{l}\text { MURAKAMI, } \\
\text { R.; CAMPOS, C. } \\
\text { J. G.; }\end{array}$ & $\begin{array}{l}\text { Analisar a } \\
\text { influência da } \\
\text { relação }\end{array}$ & $\begin{array}{l}\text { Pesquisa } \\
\text { qualitativa }\end{array}$ & $\begin{array}{l}\text { As entrevistas } \\
\text { refletiram que a falta } \\
\text { de atenção e }\end{array}$ \\
\hline $\begin{array}{l}\text { do enfermeiro } \\
\text { com a família } \\
\text { de crianças } \\
\text { hospitalizadas" } \\
\text { S2 - } 2\end{array}$ & Brasil (2011) & $\begin{array}{l}\text { interpessoal } \\
\text { entre o } \\
\text { enfermeiro e a } \\
\text { família na } \\
\text { recuperação da } \\
\text { criança } \\
\text { hospitalizada. }\end{array}$ & & $\begin{array}{l}\text { informação relativa à } \\
\text { evolução da doença } \\
\text { da criança, resulta } \\
\text { em sentimentos de } \\
\text { sofrimento, } \\
\text { impotência e } \\
\text { desprezo para o } \\
\text { familiar, podendo } \\
\text { resultar em atitudes } \\
\text { agressivas, de revolta } \\
\text { e insatisfação. }\end{array}$ \\
\hline $\begin{array}{l}\text { "Parents" } \\
\text { satisfaction } \\
\text { concerning } \\
\text { their child"s } \\
\text { hospital care" } \\
\text { S2 - 6 }\end{array}$ & $\begin{array}{l}\text { MATZIOU, V.; } \\
\text { BOUTOPOULO } \\
\text { U, B.; } \\
\text { CHRYSOSTOM } \\
\text { OU, A.; } \\
\text { VLACHIOTI, E.; } \\
\text { MANTZIOU, T.; } \\
\text { PETSIOS,K. } \\
\text { Japão (2006) }\end{array}$ & $\begin{array}{l}\text { Explorar a } \\
\text { satisfação dos } \\
\text { pais acerca dos } \\
\text { cuidados } \\
\text { durante a } \\
\text { hospitalização } \\
\text { da criança e os } \\
\text { seus } \\
\text { determinantes. }\end{array}$ & $\begin{array}{l}\text { Estudo não } \\
\text { experimenta } \\
1 \text {, } \\
\text { correlaciona } \\
1 \text { descritivo. }\end{array}$ & $\begin{array}{l}\text { A maioria dos pais } \\
\text { mostrou-se mais } \\
\text { satisfeita quando o } \\
\text { nível de informação, } \\
\text { o tratamento e o } \\
\text { comportamento da } \\
\text { equipa foi ótimo. }\end{array}$ \\
\hline $\begin{array}{l}\text { "Parents" } \\
\text { experiences of } \\
\text { participation } \\
\text { in the care of } \\
\text { hospitalised } \\
\text { children: A } \\
\text { qualitative } \\
\text { study" } \\
\mathbf{S 4 - 2}\end{array}$ & $\begin{array}{l}\text { LAM, L. W.; } \\
\text { CHANG, A.M., } \\
\text { MORRISSEY,J. } \\
\text { Japão (2006) }\end{array}$ & $\begin{array}{l}\text { Descrever a } \\
\text { experiência dos } \\
\text { pais relativas } \\
\text { aos cuidados às } \\
\text { crianças } \\
\text { hospitalizadas }\end{array}$ & $\begin{array}{l}\text { Estudo } \\
\text { Qualitativo }\end{array}$ & $\begin{array}{l}\text { Foram identificadas } \\
\text { quatro dimensões } \\
\text { fundamentais } \\
\text { associadas à } \\
\text { participação dos pais } \\
\text { nos cuidados da } \\
\text { criança } \\
\text { hospitalizada: razão } \\
\text { para ficar com a } \\
\text { criança; } \\
\text { reorganização das } \\
\text { rotinas da família; } \\
\text { expectativas em } \\
\text { relação aos } \\
\text { enfermeiros e } \\
\text { comentários sobre } \\
\text { condições das } \\
\text { instalações }\end{array}$ \\
\hline
\end{tabular}

\section{DISCUSSÃO}

Após a análise dos estudos selecionados, tornou-se claro que todos os autores enfatizam a importância da satisfação das necessidades apresentadas pelos pais ao longo do processo de hospitalização.

A informação cedida pela equipa de enfermagem aos pais torna-se essencial para promover e facilitar a participação dos mesmos nos cuidados à criança, sendo esta opinião partilhada por todos os autores.

A falta de atenção e de informação referente à evolução do estado de saúde da criança, pode despoletar sentimentos de sofrimento, desprezo e impotência por parte do familiar, podendo desencadear atitudes agressivas, de revolta e de insatisfação por parte destes (Murakami e Campos, 2011).

Apesar dos ensinos realizados e da informação transmitida pelos enfermeiros aos familiares, é necessário definir o nível de envolvimento dos pais, visto que nem todas intervenções deverão ser desempenhadas pelos mesmos, podendo levar à negligência e, consequentemente à desconfiança por parte da equipa médica relativamente à equipa de enfermagem, ideia defendida por Vasli e Salsali (2014).

Murakami e Campos (2011), defendem que os enfermeiros têm que reunir competências para estabelecer os limites de participação dos familiares, pelo que, quando existe um comprometimen- 
to da evolução do estado de saúde da criança, deve ser limitado o envolvimento dos pais. Para a melhor forma de atuação, o discurso desrespeitoso e autoritário deve ser evitado pelo profissional, procurando uma comunicação assertiva e compreensiva para o familiar. Este aspeto remete-nos para o modelo de parceria de cuidados recomenda flexibilidade nos cuidados, não preconizando a existência de fronteiras fixas (S. M. G. Santos, 2012).

0 estabelecimento de normas que permitam a permanência de um dos familiares 24 horas por dia, mas também a existência de condições a nível do espaço físico defendida por S. M. G. Santos (2012) associada aos recursos humanos e físicos, identifica como essencial para os pais que 0 ambiente hospitalar esteja devidamente equipado para a sua permanência, oferecendo um ambiente confortável e privacidade.

A presença dos pais proporciona à criança suporte emocional, permitindo que esta se sinta mais segura e menos ansiosa, diminuindo o sofrimento causado pelo processo de hospitalização (Murakami e Campos, 2011 e Lam, Chang e Morrissey, 2006). Andrade et al. (2012), consideram a família como uma "referência de vida fora do hospital", pelo que acompanhamento ao longo do tratamento, incluindo os procedimentos invasivos, contribui para a obtenção de melhores resultados e maior efetividade, diminuindo assim o impacto da hospitalização. A importância da presença dos pais na realização de procedimentos hospitalares é realçada, uma vez que, o medo causado na criança é atenuado, transmitindo segurança e um sentimento familiar (Murakami e Campos, 2011). Demonstram ainda preferência em permanecer juntamente com os seus filhos no hospital, ao invés de estarem ausentes, e estarem constantemente a pensar como é que os seus filhos estão (Lam, Chang e Morrissey, 2006).

Os pais demonstraram preocupação pela possibilidade dos enfermeiros não serem capazes de identificar todas as necessidades dos seus filhos, e de realizar os cuidados como no domicílio, mostrando-se interessados em prestar os cuidados aos seus filhos durante o processo de hospitalização (Lam, Chang e Morrissey, 2006). Vasli e Salsali (2014), concluem que a oportunidade de participação dos pais nos cuidados apresenta efeitos positivos.

0 envolvimento da família nos cuidados, o conhecimento da evolução da criança e as orientações seguidas, leva a que os pais acreditem que podem participar nos cuidados (Andrade et al., 2012), leva a que os enfermeiros partilhem os seus conhecimentos e realizem ensinos aos familiares para que estes se sintam capazes de participar no processo de cuidados (Cardoso, 2010). Para o familiar participar nos cuidados, é fundamental que este demonstre interesse e empenho, sentindo-se responsável pelos mesmos e, fornecendo suporte emocional à criança (Murakami e Campos, 2011). Participando ativamente o familiar sente-se mais útil, esquecendo a ideia de observador passivo durante 0 internamento.

Com a participação ativa nos cuidados através da realização dos procedimentos, esta permite também a aquisição de competências para os cuidados a serem realizados no domicílio após a alta (Andrade et al., 2012). Torna-se essencial a explicação dos procedimentos antes da sua concretização e 0 treino para a preparação para a alta (Murakami e Campos, 2011).

\section{CONCLUSÃO}

Relativamente ao impacto positivo para a família, concluímos que a aplicação deste modelo permite: que os familiares se sintam mais satisfeitos e capazes para a realização dos cuidados, diminuem os sentimentos de ansiedade gerados pela hospitalização de um familiar, aumentando o sentimento de independência e utilidade durante 0 internamento. A diminuição destes sentimentos negativos facilita a adaptação à nova condição de saúde da criança e capacita o familiar no que diz respeito à prestação de cuidados a realizar após a alta hospitalar. 


\section{IMPACTO DA PARCERIA DE CUIDADOS PARA A CRIANÇA HOSPITALIZADA E SUA FAMÍLIA}

Relativamente à criança, foi possível concluir que a implementação da parceria de cuidados apresenta vários benefícios: diminuição da ansiedade, medo, sofrimento e dor, através do aumento da segurança pela presença de um familiar. 0 facto da criança sentir os seus familiares mais seguros e menos ansiosos, acaba por ter repercussões a nível do seu bem-estar, uma vez que também se vai sentir mais segura e menos ansiosa. Desta forma, podemos concluir, que sendo o modelo da parceria de cuidados centrado na satisfação das necessidades da criança e da sua família, ao satisfazer as necessidades do familiar, estamos também a satisfazer as necessidades da criança hospitalizada, uma vez que estas são influenciadas pelas dos seus familiares e vice-versa.

No que diz respeito ao impacto negativo que o modelo de pareceria de cuidados pode apresentar, apenas identificamos aspectos relacionados com os familiares da criança hospitalizada. Assim, a implementação deste modelo, pode levar ao surgimento de sentimentos de stress e de sensação de incapacidade, provocados pela participação na prestação dos cuidados à criança.

Podemos assim concluir que a implementação do modelo de parceria de cuidados, contribui consideravelmente para tornar a experiência do internamento mais positiva para a criança e sua família recomendando a sua implementação em todos os hospitais pediátricos, dado que 0 impacto é consideravelmente positivo para todos os intervenientes. Assim é fundamental que os enfermeiros se mostrem disponíveis para a partilha de informação e conhecimento, recorrendo a uma comunicação clara e eficiente e, a um discurso esclarecedor, transmitindo informação relativa à hospitalização da criança de forma a promover a relação empática entre o enfermeiro e a família que facilita consideravelmente todo o processo de parceria de cuidados. É ainda crucial que o enfermeiro demonstre disponibilidade e esteja presente durante a realização dos procedimentos pelos familiares, de forma a transmitir sentimentos de segurança e realizar ensinos pertinentes quando a situação 0 exija.

\section{REFERÊNCIAS BIBLIOGRÁFICAS}

Cardoso, A. C. (2010). Experiências dos pais na hospitalização da criança com doença aguda. (Dissertação de mestrado). Recuperado de http://hdl.handle.net/10216/26863

Nascimento, L. C., Furquim, P. de S., Rigotti, A. R., Luiz, F. M., Bortoli, P. S., \& Gianoti, S. (2006). A utilização do lazer como estratégia para integração de familiares/acompanhantes em Enfermaria de Pediatria. Escola Anna Nery Revista de Enfermagem, 10(3). 580-585. doi: 10.1590/S141481452006000300031

Oliveira, G. F., Dantas, F. D., \& Fonsêca, P. N. (2004). 0 impacto da hospitalização em crianças de 1 a 5 anos de idade. Revista da Sociedade Brasileira de Psicologia Hospitalar, 7(2). 37-54. Recuperado de http://pepsic.bvsalud.org/pdf/rsbph/v7n2/v7n2a05.pdf

Santos, S. M. (2012). As necessidades dos pais da criança hospitalizada. (Dissertação de mestrado). Recuperado de http://hdl.handle.net/10314/1319

Valverde, D. L. (2010). 0 suporte psicológico e a criança hospitalizada: 0 impacto da hospitalização na criança e em seus familiares. Psicologia.pt - Portal Dos Psicólogos. Recuperado de http://www.psicologia.pt/artigos/textos/TL0229.pdf 